\title{
Locating a Vehicle Return Center via Artificial Fish Swarm Algorithm
}

\author{
Qiang $\mathrm{Li}^{1, \mathrm{a}}$, Hongfei Jia ${ }^{1, \mathrm{~b}^{*}}$, Hongliang $\mathrm{Li}^{2, \mathrm{c}}$ and $\mathrm{Yu} \mathrm{Lin}^{2, \mathrm{~d}}$ \\ ${ }^{1}$ Transportation College, Jilin University, Changchun 130022, China \\ 2 Transportation College, Northeast Forestry University, Harbin 150040, China \\ acidiyi@163.com, bjiahf@jlu.edu.cn, 'Ihliang@nefu.edu.cn, dinyu773@163.com \\ ${ }^{*}$ corresponding author: Hongfei Jia
}

Keywords: Sustainable facility location allocation, Optimization Algorithm, Modeling and simulation

\begin{abstract}
Transportation facility or automotive service enterprise location is an interesting and important issue. To improve transportation efficient, lots of researchers have addressed traditional facility location allocation (FLA) problem, e.g., the FLA problem with the minimum transportation cost or the maximum obtained profit. This work aims to optimize obsolete vehicle location with the minimum transportation cost. However, the return center location on obsolete vehicle is influenced by regional constraints. Thus, the regional constraint should be considered as factors influencing the FLA problem. To handle this issue via a more practical method, this work proposes an economic optimal model with region constraints for an automotive service enterprise. That is, by taking the vehicle return center as a typical automotive service enterprise and an example, this work presents its new economic optimal models considering region and non-region constraints. An artificial fish swarm algorithm is proposed to solve the proposed models. Some numerical examples are given to illustrate the proposed models and testify the effectiveness of the algorithm.
\end{abstract}

\section{Introduction}

Since facility location allocation (FLA) problems were initialized by Cooper [1] in 1963, there have been many advances in their solution methods, variants and applications, e.g., emergency service systems, telecommunication networks, public services, and transportation facilities. The early work mainly focused on FLA location problems, many different models have been formed [1-4], i.e., median models [5-6], center models [7-10], covering models [11-12], hub location models [13], and hierarchical location models [14-16]. Meanwhile, a large number of solution approaches for different models have been proposed, e.g., simulated annealing, Genetic Algorithm, and Tabu Search [17-25].

The location of an automotive service enterprise is one of the important applications of FLA. Some scholars have discussed the related issues under uncertainty. For example, by considering the uncertainty of the number of inspection vehicles, Tian et al. established a stochastic location model for vehicle inspection station to achieve the minimum transportation cost of vehicle inspection customers [26]. They proposed a hybrid algorithm integrating Genetic Algorithm (GA) and Neural networks (NN) to solve this vehicle inspection station model [27]. By considering the uncertainty of the number of inspection vehicles and road conditions under different districts of inspection customers, they proposed the expected value model for vehicle inspection station location problem with random inspection demand and different transportation cost [28]. Tian et al. established a cost-profit tradeoff model for a vehicle inspection station with stochastic inspection demand [29]. Ling et al. solved the car parking location problem to minimize the total transportation cost of customers based on an iterative algorithm [30]. Wang et al. analyzed the location and pricing of a park-and-ride facility under alternative profit maximization and social cost minimization objectives [31].

Based on the above overview, for automotive service enterprise location, the researchers mainly focus on the vehicle inspection station, but they pay little attention to the vehicle return center location issue. Thus, this work proposes the location problem for vehicle return center. Moreover, in the actual location process, some regions cannot build the service facility due to the influence of 
government policy and natural factors, i.e., marshes, lakes, tourist areas, parks, residential areas and some special areas with the policy constraints. These regions should be considered when they are planned. To do so, by taking a return center as a typical automotive service enterprise and an example, this work proposes to establish economic optimal models of its location considering region and non-region constraints for the first time to the best knowledge of the authors.

In Section II, the assumptions and parameters of proposed models are introduced. In Section III, models of location for a return center are established. Section IV introduces the algorithm to solve these models. In Section V, some numerical examples are presented to test the effectiveness of the proposed algorithm. Finally, Section VI concludes our work and describes some future research issues.

\section{Problem Statements}

In order to establish our models conveniently, the following assumptions and parameters are given in this work.

A. Parameters

(1) $i$-The index of obsolete vehicle demand region.

(2) $j$-The index of need to establish a return center.

(3) $\left(l_{i}, u_{i}\right)$-The coordinate of the location of the $i$ th obsolete vehicle demand region.

(4) $\left(x_{j}, y_{j}\right)$-The coordinate of the location of the $j$ th a return center. Note that it is a decision variable in this work.

(5) $c_{i j}$-Per kilometer transport consumption from obsolete vehicle demand region $i$ to a return center $j$, with its unit being yuan.

(6) $a_{i j}$-The number of vehicles from obsolete vehicle demand region $i$ to a return center $j$.

B. Assumptions

This work makes the following assumptions:

(1) The distribution condition of obsolete vehicle demand region is not taken into consideration and the center of obsolete vehicle demand region is treated as the coordinate location of obsolete vehicle demand region .

(2) The location of obsolete vehicle is generally counted by the regional statistics.

(3) The cost per kilometer $c_{i j}$ from obsolete vehicle demand region $i$ to a return center $j$ is constant.

(4) The return center capability of a vehicle inspection station is ignored, namely return center is big enough to meet requirement of users.

\section{Economic Optimal Model Of The Location For A Return Center}

In the process of the location of the obsolete vehicle return center, if the target is different, the programming model is also different. This work takes the lowest total transportation cost of customer as the goal of the location, the following model can be constructed:

$$
\min \mathrm{C}
$$

where $C$ is the total transport cost of a return center customers and it is expressed as $\min C=\sum_{i} \sum_{j} a_{i j} c_{i j} d_{i j} . a_{i j}$ is the number of vehicles from obsolete vehicle demand region $i$ to a return center $j$, and $d_{i j}$ is the distance between obsolete vehicle demand region $i$ and a return center $j$ and it is expressed as $d_{i j}=\sqrt{\left(x_{j}-l_{i}\right)^{2}+\left(y_{j}-u_{i}\right)^{2}} \cdot x_{l}$ and $x_{u}$ are the lower and upper bounds of the coordinate $x$, respectively, $y_{l}$ and $y_{u}$ are the lower and upper bounds of coordinate $y . x_{l}, x_{u}, y_{l}$ and $y_{u}$ can be determined by the coordinate of obsolete vehicle demand regions .

But in the actual location of the process, the swamps, lakes, tourist areas, parks and residential 
areas may be encountered. The above areas are unable to carry out the construction work of return center. thus, when it is planned, these areas should be excluded in advance. In order to solve the problem of return center selection in this type of region, it is necessary to introduce a planning model with region constraints. The return center location planning model with regional constraint is expressed as follows:

$$
\min C=\sum_{i} \sum_{j} a_{i j} c_{i j} d_{i j}
$$

Subject to:

$$
\left\{\begin{array}{l}
h(x, y) \leq 0 \\
g(x, y) \geq 0
\end{array}\right.
$$

where $h(x, y) \leq 0, g(x, y) \geq 0$ are regional constraint conditions.

\section{Solution Algorithm}

Artificial fish swarm algorithm (AFSA) is an effective method to solve industrial optimization problems, e.g., wastewater management, clustered oversubscription planning and nuclear fuel cycle [32-34]. Thus, we propose to adopt artificial fish swarm algorithm to solve the established models. Its basic ideas are presented next.

\section{A. AFSA}

According to the swarm intelligence, AFSA is an artificial intelligent algorithm based upon the simulation of the collective behaviors of fish stocks. It simulates the behavior of an artificial fish $(A F)$, and then constructs a swarm of $A F$. Every one of $A F$ will search its own best of local, pass on information in its self-organized system and finally achieve the best of global. We should initialize the school of fish randomly. Let the searching space be $D$-dimensional and there are Fishnum fishes in the colony. The current state vector of an $A F$ swarm is $Z=\left(z_{1}, z_{2}, \ldots, z_{m}\right)$, where $z_{i}(i=1, \ldots, m)$ is the variable to be optimized. Visual is the visual distance. The $A F$ occurs only in the inner radius of the circle to the length of the field of vision various acts. The foodconsistence of $A F$ in the current position is represented by $Y=F(Z)$, where $Y$ is the objective function. The distance between the ith and the $j$ th individual $A F$ can be expressed as $d_{i j}=\left\|Z_{j} Z_{i}\right\|, i$ and $j$ are random fishes. Step means the maximum step size of $A F . \rho(0<\rho<1)$ is the degree of congestion factor. In the initial state of the algorithm, the iterative number of the variable should be defined as iterative times of $A F$ searching for food. In addition, Maxgen is set as the limitation of iterative times in this work. Because the maximization problem and the minimization problem can be transformed with each other, we take the maximization problem as an example in the following analysis.

1) Preying behavior

This is a basic biological behavior that tends to the food. Let the current state of an $A F$ be $Z_{i}$, then we select a new state $Z_{j}$ randomly in its visual field. If the foodconsistence of $Z_{j}$ is bigger than that of $Z_{i}$, it moves a step in that direction, otherwise, we select a state $Z_{j}$ randomly again and judge whether it satisfies the forward condition. If it can not be satisfied after a preset Try_number times, it moves a step randomly. The step moving follows the following rule:

$$
\begin{cases}Z_{i+1}=Z_{i}+\text { Step } \times \frac{Z_{j}-Z_{i}}{\left\|Z_{j}-Z_{i}\right\|} \times \operatorname{rand}() & \left(Y_{j}>Y_{i}\right) \\ Z_{j}=Z_{i}+\text { Visual } \times \text { rand }() & \left(Y_{j}<Y_{i}\right)\end{cases}
$$

where rand () is a random number and ranges 0 from 1 .

2) Swarming behavior

Let $Z_{i}$ be the current state of $A F$ searching companion $Z_{\max }$ in the neighborhood with $Y_{\max }$, if $Y_{\text {max }} / n_{f}>\rho Y_{i}$, it means the current position of companion $Z_{\text {max }}$ has higher food consistence and it is not crowded. The $A F$ will move a step towards companion $Z_{\max }$; otherwise, continue searching behavior. The following behavior is mathematic description: 


$$
\begin{cases}Z_{i+1}=Z_{i}+\operatorname{Step} \times \frac{Z_{c}-Z_{i}}{\left\|Z_{c}-Z_{i}\right\|} \times \operatorname{rand}() & \left(\frac{Y_{c}}{n_{f}}>\rho Y_{i} \text { and } n_{f} \geq 1\right) \\ Z_{i+1}=\text { Formula(5) } & \left(\frac{Y_{c}}{n_{f}} \leq \rho Y_{i} \text { or } n_{f}=0\right)\end{cases}
$$

3) Following behavior

Let $X_{i}$ be the current state of $A F$ and search its companion $Z_{\max }$ whose $Y_{\max }$ is the largest in the neighborhood, if $Y_{\max } / \mathrm{n}_{\mathrm{f}}>\rho Y_{i}$, it means that the current position of companion $Z_{\max }$ has higher food consistence and it is not crowded. The $A F$ would move a step towards companion $Z_{\text {max }}$, otherwise, the preying behavior would be executed.

The following is mathematic description:

$$
\left\{\begin{array}{cl}
Z_{i+1}=Z_{i}+\text { Step } \times \frac{Z_{\max }-Z_{i}}{\left\|Z_{c}-Z_{i}\right\|} \times \text { rand }() & \left(\frac{Y_{\max }}{n_{f}}>\rho Y_{i} \text { and } n_{f} \geq 1\right) \\
Z_{i+1}=\text { Formula }(5) & \left(\frac{Y_{\max }}{n_{f}} \leq \rho Y_{i} \text { or } n_{f}=0\right)
\end{array}\right.
$$

4) Bulletin

Bulletin is used to record the optimal result of $A F$ and the optimal value of the problem. Each $A F$ updates its own state and compares it with that on the bulletin after making movements. If its current state of $A F$ is better, then the value on the bulletin would be replaced.

Based on the above presentation, AFSA has the following steps.

Step 1) Initialize the parameters of artificial fish, i.e., Step and Visual, the maximum number of exploration Try_number, the maximum number of iterations Maxgen, and the number of fishes Fishnum. Note that the carbon emission constraints should be checked in this Step.

Step 2) Set bulletin board to record the current status of each fish, and select the optimal value record.

Step 3) Implement preying, swarming and following behaviors.

Step 4) Update the optimal value on the bulletin board.

Step 5) If the Maxgen is accomplished, the optimal result would be output, otherwise AFSA returns to Step 2.

The above algorithm has been implemented in the MATLAB programming language (R2010b).

\section{Case Study}

Considering a location problem of the vehicle return center for Fushun in China, it will be applied to our proposed models. This city is divided into five obsolete vehicle regions, i.e., Development, Dongzhou, Wanghua, Xinfu and Shuncheng districts, as shown in Fig. 1. Their central coordinates of each district are listed in Table I. In addition, the central coordinate of this city is (41578784.96, 4638592.97). In order to optimize the location problem conveniently, taking the center coordinate of this city as relative coordinate origin, and the coordinates of these five districts are transformed into the following numerical values, as shown in Table 1 . In addition, return rate and the cost consumption per kilometer of each demand region are listed in Table 1.

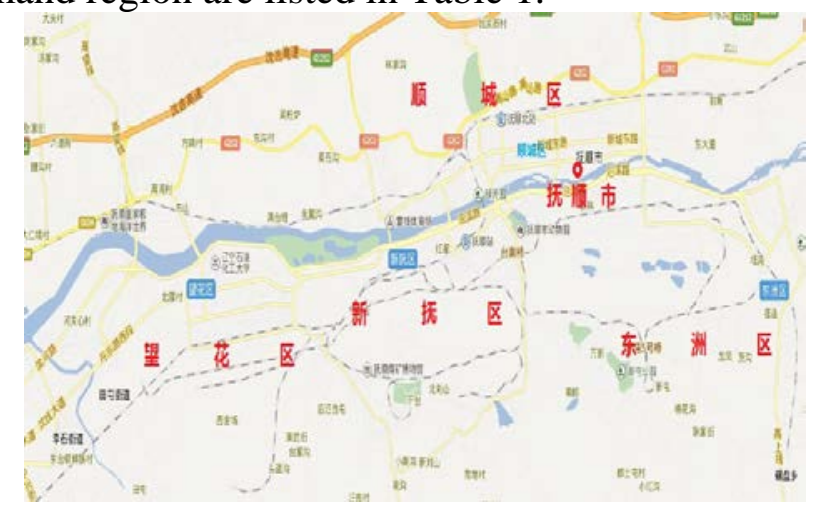

Fig. 1. Schematic diagram of Fushun city 
Table 1 Typical parameters return center of established model

\begin{tabular}{|c|c|c|c|c|c|c|}
\hline Region No. & Region Name & $l(\mathrm{~m})$ & $u(\mathrm{~m})$ & $a$ (vehicle) & rate $(\%)$ & $C$ (yuan) \\
\hline 1 & Dongzhou & 6818.23 & -2988.68 & 2884 & 4 & 2 \\
\hline 2 & Xinfu & -3625.74 & -2088.84 & 3556 & 4 & 2 \\
\hline 3 & Wanghua & -14319.44 & -3175.23 & 1557 & 4 & 2 \\
\hline 4 & Shuncheng & -1109.74 & 285.12 & 5074 & 4 & 2 \\
\hline 5 & Development & -19553.93 & -6822.91 & 2660 & 4 & 2 \\
\hline
\end{tabular}

The parameters of the AFSA are set next: the number of fish Fishnum is 50, the visual distance Visual is 1, the Step is 0.1, the maximum number of generations Maxgen is 100, the degree of congestion factor $\rho$ is 0.618 , and the number of exploration Try_number is 100 .

Example 1. A decision-maker hopes to build a return center with the minimum transport consumption of obsolete vehicle customers. This problem can be translated to solve the following model:

$$
\min \mathrm{C}
$$

Subject to:

$$
\left\{\begin{array}{l}
x \in(-19553.93,6818.23) \\
y \in(-6822.87,285.12)
\end{array}\right.
$$

After the algorithm is executed, the following results can be obtained:

$$
\begin{aligned}
(x, y) & =(-2661.18,-1049.46) \\
\mathrm{C} & =8.74 \times 10^{3} .
\end{aligned}
$$

The results denote that the location coordinate of the return center is $(-2661.18,-1049.46)$, the lowest total transportation cost of obsolete vehicle customers is $8.74 \times 10^{3}$ Yuan.

In addition, the plane distribution graph for the location of a vehicle return center is obtained and shown in Fig.2.

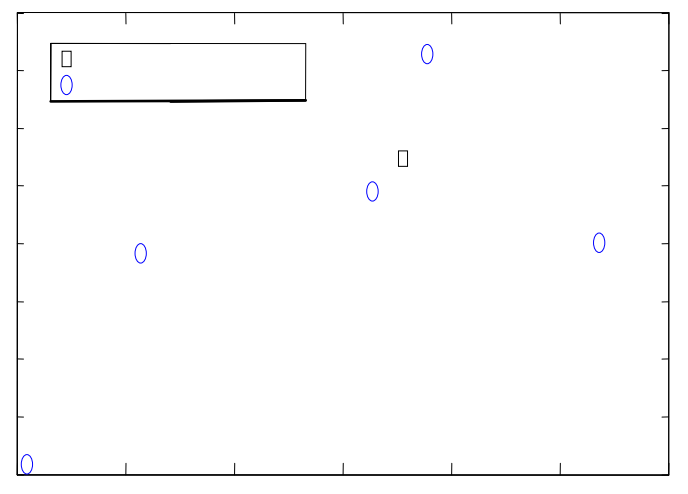

Fig. 2. Plane distribution graph for the location of a return center

Example 2. A decision-maker hopes to build a vehicle return center with the minimum transportation cost and ask to satisfy the region constraint, i.e., $x^{2}+y^{2} \geq 1.2 \times 10^{7}$. This problem can be translated to solve the following model with regional constraints:

$\min \mathrm{C}$

Subject to:

$$
\left\{\begin{array}{l}
x^{2}+y^{2} \geq 1.2 \times 10^{7} \\
x>0, y>0
\end{array}\right.
$$

After the algorithm is executed, the following results can be obtained:

$$
\begin{gathered}
(x, y)=(-2832.85,-2783.68) \\
\min C=9.04 \times 10^{3}
\end{gathered}
$$

The results denote that the location coordinate of a return center is (-2832.85,-2783.68), and the lowest total transportation cost of customers is $9.04 \times 10^{3}$ Yuan. 
Also, when the AFSA is executed, the plane distribution graph for the location of a vehicle return center is obtained and shown in Fig. 3.

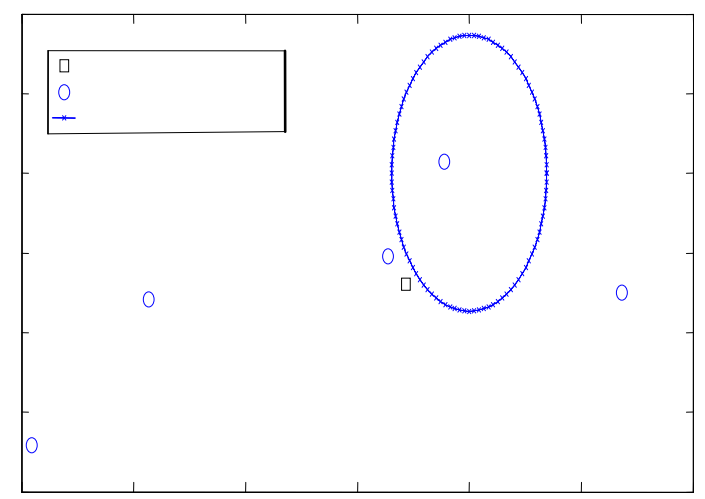

Fig.3. The graph of obsolete vehicle return center

From results and Figs. 2 to 3, we see that the optimal solutions meet given constraints and the location of vehicle return center is legitimately located. Additionally, it meets the requirement of region constraints if region any. The results denote that the proposed approach is feasible and effectively to solve the models of the location problem for a vehicle return center.

\section{Conclusions}

The location transportation facility or automotive service enterprise has a great impact on the execution of related works of economic benefits. This work aim to raise the utilization rate and economic benefits via optimizing the location of obsolete vehicle return center. To solve these problems, this paper proposes to establish economic optimal models of optimal location for an obsolete vehicle return center for the first time, i.e., economic optimal model without region constraint and economic optimal model with region constraint. In addition, an artificial fish swarm algorithm is proposed to solve the established models. The results reveal that it is feasible and effective when being used to solve the established models. The results can be used to guide decision makers in making better decisions when a vehicle return center is planned.

There are some limitations with the proposed method. For example, for the special nature constraint, this work merely considers the condition of the round shape. However, the region constraint can more likely to be irregular shapes in reality and the matter that how to deal with this issue needs to be further discussed. In addition, the uncertainty analysis and control for locating an automotive service enterprise needs further discussion [35-40].

\section{Acknowledgments}

This work is supported in part by supported in part by National Natural Science Foundation of China under Grant Nos. 51405075 and Funds for International Cooperation Exchange of the National Natural Science Foundation of China under Grant No. 51561125002.

\section{References}

[1] L. Cooper. Location-allocation problems[J]. Operation Research, 1963(11): 331-344.

[2] B. A. Murtagh and S. R. Niwattisyawong. Efficient method for the multi-depot location em dash allocation problem[J]. Journal of Operation Research Society, 1982, 33: 629-634.

[3] M. A. Badri. Combining the analytic hierarchy process and goal programming for global facility location-allocation problem[J]. International Journal of Production Economics, 1999, 62(3): 237-248. 
[4] M. Hodey, E. Melachrinoudis and X. Wu. Dynamic expansion and location of an airport: a multiple objective approach[J]. Transportation Research Part A: Policy and Practice, 1997, 31(5): 403-417.

[5] H. K. Rajagopalan, C. Saydam and J. Xiao. A multi-period set covering location model for dynamic redeployment of ambulances[J]. Computers Operation Research, 2008, 35: 814-826.

[6] R. Z. Farahani, N. Asgari, N. Heidari, M. Hosseininia, M. Goh. Covering problems in facility location: A review[J]. Computers and Industrial Engineering, 2012, 62(1): 368-407.

[7] C. Toregas, R. Swain, C. ReVelle, and L. Bergman. The location of emergency service facilities[J]. Operations Research, 1971, 19(6): 1363-1373.

[8] R. Church and C. ReVelle. The maximal covering location problem[J]. Papers of Regional Science Association, 1974, 32: 101-118.

[9] R. Z. Farahani and M. Hekmatfar. Facility location: Concepts, models, algorithms and case studies[M]. Heidelberg: Physica-Verlag, 2009.

[10] S. Nickel and J. Puetro. Location theory: A unified approach[J]. Mathematical Methods of Operations Research, 2007, 66(2): 369-371.

[11] M. S. Daskin. Network and discrete location: Models, algorithms, and applications[J]. Journal of the Operational Research Society, 1997, 48(7): 763-764.

[12] S. L. Hakimi. Optimum locations of switching centers and the absolute centers and medians of a graph[J]. Operations Research, 1964, 12(3): 450-459.

[13] M. E. O’Kelly. A quadratic integer program for the location of interacting hub facilities[J]. European Journal of Operational Research, 1987, 32(3): 393-404.

[14] A. Klose and A. Drexl. Facility location models for distribution system design[J]. European Journal of Operational Research, 2005, 162(1): 4-29.

[15] Z. Drezner and H. Hamacher. Facility location: Applications and theory[M]. Berlin: Springer-Verlag, 2004.

[16] G. Sahin and H. Sural. A review of hierarchical facility location models[J]. Computers and Operations Research, 2007, 34(8): 2310-2331.

[17] A. B. Arabani and R. Z. Farahani. Facility location dynamics: An overview of classifications and applications[J]. Computers and Industrial Engineering, 2012, 62(1): 408-420.

[18] R. E. Kuenne and R.M. Soland. Exact and approximate solutions to the multisource Weber problem[J]. Mathematical Programming, 1972, 3(1): 193-209.

[19] A. T. Murray and R. L. Church. Applying simulated annealing to location-planning models[J]. Journal of Heuristics, 1996, 2(1): 31-53.

[20] A. T. Ernst and M. Krichnamoorthy. Solution algorithms for the capacitated single allocation hub location problem[J]. Annals of Operations Research,1999, 86: 141-159.

[21] D. Gong, M. Gen, W. Xu and G. Yamazaku. Hybrid evolutionary method for obstacle location-allocation problem[J]. Computers and Industrial Engineering, 1995, 29(1): 525-530.

[22] R. Z. Farahani, M. SteadieSeifi and N. Asgari. Multiple criteria facility location problems: A survey[J]. Applied Mathematical Modelling, 2010, 34(7): 1689-1709.

[23] K. J. Wang, B. Makond and S. Y. Liu. Location and allocation decisions in a two-echelon supply chain with stochastic demand-A genetic-algorithm based solution[J]. Expert Systems with Applications, 2011, 38(5): 6125-6131.

[24] Z. Q. Lu and N. Bostel. A facility location model for logistics systems including reverse flows: The case of remanufacturing activities[J]. Computer Operation Research, 2007, 34(2): 299-323.

[25] M. A. Arostegui Jr., S. N. Kadipasaoglu, and B. M. Khumawala. An empirical comparison of Tabu Search, Simulated Annealing, and Genetic Algorithms for facilities location problems[J]. International Journal of Production Economics, 2006, 103(2): 742-754.

[26] G. D., Tian, Y. M. Liu, G. Xu, J. Y. Dai and H. G. Ren. Analysis of Network Layout Optimization Problem for Vehicle Inspection Station in Random Environment[J]. Journal of Jilin University(Engineering and Technology Edition), 2011, 41: 122-125.

[27] T. G. Qiang, G. D., Tian, J. W. Chu, Z. K. Li and D. H. Xiong. Location analysis of vehicle inspection station based on GA-NN[J]. Adv. Inf. Sci. Serv. Sci., 2013, 5: 4. 
[28] G. D. Tian, Y. M. Liu, J. Xie, Z. J. Lai and X. N. Cao. Expected Value Model for Vehicle Inspection Station Location Problem with Random Inspection Demand and Different Transportation Cost[J]. Appl. Mech. Mater., 2011, 97: 542-545.

[29] G. D. Tian, M. C. Zhou, J. W. Chu, T. G. Qiang and H. S. Hu. Stochastic cost-profit trade-off model for locating an automotive service enterprise[J]. IEEE Transactions on Automation Science and Engineering, 2014.

[30] L. Ling, W. Li, W. Wang and G. Hu. Multi-area distributing and iterative optimal location algorithm for parking areas[J]. China Civil Engineering Journal, 2003, 36(7): 18-21.

[31] J.Y.T. Wang, H. Yang and R. Lindsey. Locating and pricing park-and-ride facilities in a linear monocentric city with deterministic mode choice[J]. Transportation Research Part B, 2004, 38(8): 709-731.

[32] K. M. Wagialla, M. M. El-Halwagi and J. M. Ponce-Ortega. An integrated approach to the optimization of in-plant wastewater interception with mass and property constraints[J]. Clean Technologies and Environmental Policy, 2012, 14(2): 257-265.

[33] Z. Huang and Y. Chen. An improved artificial fish swarm algorithm based on hybrid behavior selection[J]. International Journal of Control and Automation, 2013, 6(5): 103-116.

[34] M. Neshat, G. Sepidnam, M. Sargolzaei, M. Sargolzaei, A. N. Toosi. Artificial fish swarm algorithm: a survey of the state-of-the-art, hybridization, combinatorial and indicative applications[J]. Artificial Intelligence Review, 2014, 42(4): 965-997.

[35] G. D. Tian, M. C. Zhou, J. W. Chu and Y. M. Liu. Probability evaluation models of product disassembly cost subject to random removal time and different removal labor cost[J]. IEEE Transactions on Automation Science and Engineering, 2012, 9(2): 288-295.

[36] Y. F. Fang, F. Chu and M. C. Zhou. Optimal Lane Reservation in Transportation Network[J]. IEEE Transactions on Intelligent Transportation Systems, 2012, 13(2): 482-491.

[37] W. Sun, H. Gao and B. Yao. Adaptive Robust Vibration Control of Full-Car Active Suspensions with Electro-hydraulic Actuators[J]. IEEE Transactions on Control Systems Technology, 2013, 21(6): 2417-2422.

[38] W. Sun, H. Gao and O. Kaynak. Finite Frequency Hœ Control for Vehicle Active Suspension Systems[J]. IEEE Transactions on Control Systems Technology, 2011, 19(2): 416-422.

[39] H. Ke and B. Liu. Project scheduling problem with stochastic activity duration times[J]. Applied Mathematics and Computation, 2006, 168(1): 342-353.

[40] H. Ke and B. Liu. Project scheduling problem with mixed uncertainty of randomness and fuzziness[J]. European Journal of Operational Research, 2007, 183(1): 135-147. 